\title{
Importanza del terziario per lo sviluppo regionale: il caso ticinese
}

\section{Introduzione}

Tradizionalmente, le teorie dello sviluppo regionale si fondano sul settore secondario e in particolare sullo sviluppo del settore industriale (ROSSI, 1985). Questa costatazione vale sia per i modelli dello sviluppo che mettono in rilievo il contributo della domanda globale, sia per quelli che, seguendo piuttosto le indicazioni della scuola neo-classica, sottolineano l'importanza dell'apporto dell'offerta globale e della relazione che soggiace alla funzione di produzione. Facendo questa affermazione non vogliamo sostenere che il contributo allo sviluppo del settore terziario sia stato sin qui dimenticato; intendiamo piuttosto attirare l'attenzione del lettore sul ruolo secondario che la teoria dello sviluppo regionale ha affidato, sin qui, al settore terziario. Così in modelli della domanda globale del tipo di quelli cosiddetti della «base economica», il terziario appare come il settore dipendente, le cui attività sono tipicamente locali, e quindi non in grado di riflettersi, in modo moltiplicativo, sulla crescita del reddito regionale (MERLIN, 1973). È merito, pensiamo, di POLESE di avere per primo messo in evidenza il fatto che il terziario, specie in una società di tipo post-industriale, poteva con ogni titolo essere riconosciuto come un settore motore, in grado di garantire in modo autonomo, attraverso per esempio l'esportazione dei servizi, lo sviluppo dell'economia regionale (POLESE, 1974).

Questi brevi cenni basteranno per richiamare nella sua essenza l'attualità del dibattito attorno al contributo del settore terziario allo sviluppo economico di una regione. Nel nostro contributo non intendiamo proseguire oltre nella rassegna degli aspetti teorici. Intendiamo invece presentare, con l'aiuto di un caso concreto, l'economia del Cantone Ticino, alcune linee interpretative di quello che può essere, nei fatti, l'apporto del terziario allo sviluppo di una regione. Svilupperemo l'analisi di questo caso su due sezioni: la prima di esse sarà dedicata ad un esame dell'importanza del terziario a livello cantonale, mentre la seconda tratterà di un caso particolare, ossia dello sviluppo delle attività finanziarie nella città di Lugano. Il presente contributo terminerà con una sezione conclusiva nella quale cercheremo di fare il punto della situazione in materia di relazione tra espansione del terziario e sviluppo dell'economia regionale.

\section{Il contributo del terziario a livello cantonale}

Nelle versioni più tradizionali del modello della base economica le attività del terziario sono designate come «non basic», ossia non autonome e vengono fatte dipendere dall'aumento della popolazione, applicando regole di calcolo che ricordano vagamente i rapporti di popolazione minima con i quali, nella pratica pianificatoria, si è cercato di dare un contenuto concreto alla gerarchia delle località centrali (CHRISTALler, 1933). Osserviamo che nel caso del Cantone Ticino l'evoluzione della popolazione non sembra rappresentare un buon indicatore per spiegare l'espansione dell'occupazione nel settore terziario. Dal 1950 al 1980, infatti, la popolazione residente di questo Cantone è aumentata del $51,8 \%$; nel medesimo periodo di tempo, l'occupazione nel settore terziario della sua economia è più che raddoppiata. Se l'aumento di popolazione è considerato come un'approssimazione all'aumento del consumo interno di servizi, questa variabile non basterebbe a spiegare la totalità dell'aumento dell'occupazione del terziario. È probabile, anzi verosimile, che all'aumento della domanda interna occorra aggiungere un consistente apporto di domanda esterna. Che i servizi di un'economia regionale non siano destinati unicamente a soddisfare una domanda di origine interna lo si percepisce abbastanza rapidamente nei casi in cui la domanda esterna è soddisfatta all'interno del territorio regionale. L'esempio delle attività del settore turistico è forse quello più caratteristico. Di fatto, tuttavia, non esiste nessuna preclusione in materia di esportazione di servizi. Come sostengono taluni autori anche un settore apparentemente orientato verso i bisogni della popolazione locale, come il settore pubblico, deve essere considerato, a livello di una

Angelo Rossi, Prof., Dr., Institut de Hautes Etudes en Administration Publique (IDHEAP), Av. de Provence 22, 1007 Lausanne 
regione, come un settore di esportazione, nella misura in cui occupi delle persone che appartengono ad amministrazioni, come quella federale, la cui sede è esterna alla regione (ISSERMAN, 1980).

Un metodo grezzo ma abbastanza informativo per determinare la parte di un settore o di un ramo che è orientata verso l'esportazione e che quindi contribuisce in modo autonomo allo sviluppo dell'economia regionale, è quello del coefficiente di localizzazione. Ricorrendo, a causa della disponibilità di dati, all'impiego come variabile di riferimento, il coefficiente di localizzazione per ogni ramo del terziario, può essere determinato, utilizzando la seguente formula:

$$
\mathrm{CL}_{\mathrm{ir}}=\frac{\mathrm{O}_{\mathrm{ir}} / \mathrm{O}_{\mathrm{r}}}{\mathrm{O}_{\mathrm{in}} / \mathrm{O}_{\mathrm{n}}}
$$

Nell'equazione (1), $\mathrm{CL}_{\mathrm{ir}}$ sta per coefficiente di localizzazione del ramo i nella regione $\mathrm{r}, \mathrm{O}_{\mathrm{ir}}$, per occupazione nel ramo i della regione $\mathrm{r}$ e $\mathrm{O}_{\mathrm{r}}$ per il totale dell'occupazione nella regione $r$. Analogamente $\mathrm{O}_{\text {in }} \mathrm{e}$ $\mathrm{O}_{\mathrm{n}}$ rappresentano l'occupazione nel ramo i e il totale dell'occupazione a livello nazionale.

La quota dell'occupazione di un ramo che può essere considerata come orientata verso l'esportazione viene determinata dall'equazione seguente:

$$
E_{i r}=\left(1-1 / C L_{i r}\right) \cdot O_{i r}
$$

ISSERMAN dimostra che questa equazione è equivalente alla seguente:

$$
\text { (3) } E_{\text {ir }}=\left(O_{\text {ir }} / O_{\text {in }}-O_{r} / O_{n}\right) \cdot O_{\text {in }}
$$

Nell'equazione (3) i due rapporti tra parentesi rappresentano rispettivamente la quota di produzione di un ramo regionale nel totale nazionale $\left(\mathrm{O}_{\mathrm{ir}} / \mathrm{O}_{\text {in }}\right)$ e la quota di consumo di una regione nel totale del consumo nazionale $\left(\mathrm{O}_{\mathrm{r}} / \mathrm{O}_{\mathrm{n}}\right)$. Questa approssimazione è accettabile a condizione che si reputi che il livello di produttività della regione non sia diverso da quello medio nazionale e che la quota dei consumi sia uguale nella regione come a livello nazionale. Nel quadro di queste condizioni - abbastanza rigide come si può costatare - la differenza tra i due rapporti in parentesi può essere considerata come una misura dell'eccesso di produzione regionale rispetto al consumo del mercato interno, ossia come una misura della quota di esportazione del ramo.

Con i dati relativi alla popolazione occupata, derivati dal censimento federale della popolazione, abbiamo potuto calcolare il coefficiente di localizzazione per i rami che compongono il settore terziario, utilizzando la formula dell'equazione (1).

Nella tabella 1 sono riportati i valori del coefficiente di localizzazione per ramo e per l'insieme del settore terziario nel 1950 e nel 1980.
Tab. 1 Coefficienti di localizzazione per i rami del terziario del Cantone Ticino, nel 1950 e nel 1980

\begin{tabular}{|l|r|r|r|}
\hline Rami e settore & \multicolumn{3}{|c|}{ Coefficienti di localizzazione } \\
& 1950 & 1980 & Variazione \\
\hline 1. Commercio & 0,92 & 0,96 & $+0,04$ \\
2. Banche, Assicurazioni, & & & \\
$\quad$ Mediazioni & 1,29 & 1,59 & $+0,30$ \\
3. Trasporti, Comunicazione & 1,56 & 1,43 & $-0,13$ \\
4. Industria alberghiera & 1,21 & 1,46 & $+0,25$ \\
5. Amministr. pubblica, & & & \\
$\quad$ insegnamento, salute & 0,72 & 1,05 & $+0,33$ \\
6. Altri servizi & 1,18 & 1,23 & $+0,05$ \\
\hline \multicolumn{1}{|c|}{ Settore terziario } & 1,07 & 1,19 & $+0,12$ \\
\hline
\end{tabular}

Fonte: Elaborazione dell'autore

Con due eccezioni nel 1950 e con una sola eccezione nel 1980, i rami del terziario ticinese possiedono tutti un coefficiente di localizzazione superiore a 1. Si tratta quindi di rami che non producono servizi per il solo mercato locale, ma che esportano, in misura minore o maggiore, una parte della loro produzione contribuendo così, in modo autonomo all'espansione dell'economia regionale. I valori dei coefficienti di localizzazione ci permettono di accertare quali sono state le trasformazioni importanti nella struttura del terziario ticinese, dal 1950 al 1980. Mentre all'inizio del periodo considerato il ramo esportatore per eccellenza era quello delle comunicazioni e dei trasporti, alla fine del periodo il coefficiente di localizzazione più elevato si ritrova nel ramo delle banche, delle assicurazioni e dei servizi di mediazione, ramo che chiameremo, per semplificare il riferimento «ramo dei servizi finanziari». L'avanzamento più importante, tuttavia, non è quello manifestatosi nel ramo dei servizi finanziari, ma quello manifestatosi nel ramo dei servizi pubblici, che riunisce l'amministrazione pubblica, l'insegnamento e i servizi della salute. Perdente, in termini di coefficiente di localizzazione, è il ramo dei trasporti e delle comunicazioni. È importante, dal profilo di un giudizio sul contributo dei diversi rami allo sviluppo dell'economia regionale, ricordare che in parte la variazione del coefficiente di localizzazione, dall'inizio del periodo alla fine dello stesso, è legata all'evoluzione della produttività del ramo. Sappiamo così che nel ramo dei trasporti e delle comunicazioni (DUPASQUIER, 1985) la produttività è aumentata a un ritmo sostenuto, riducendo verosimilmente il fabbisogno di forza-lavoro. All'estremo opposto si trova il ramo dei servizi pubblici nel quale, durante il periodo considerato, la produttività non è aumentata in modo considerevole. Ovviamente queste non possono essere che considerazioni generali in quanto per un'epurazione dei coefficienti di localizzazione dalla loro componente di produttività occorrerebbe poter disporre di indica- 
zioni sulla produttività per ramo che, per il momento, non si ritrovano nella statistica economica svizzera.

Utilizzando l'equazione (2) e i dati della tabella 1, possiamo calcolare, in termini assoluti e come percentuale dell'impiego del ramo, il volume di manodopera orientato verso l'esportazione. I risultati di questa stima, per l'anno iniziale e finale del periodo considerato, sono riportati nella tabella 2 .

Tab. 2 Volume di manodopera orientata verso l'esportazione per ramo, nel 1950 e nel 1980

\begin{tabular}{|l|r|r|r|r|}
\hline Ramo e settore & \multicolumn{4}{|c|}{ Mandopera orientata } \\
& \multicolumn{2}{|c|}{1950} & \multicolumn{2}{c|}{1980} \\
& in unità & in \% & in unità & in \% \\
\hline 1. Commercio & & & & \\
2. Servizi finanziari & 413 & $22,5 \%$ & 3262 & $37,1 \%$ \\
3. Trasporti, Comunic. & 1962 & $35,9 \%$ & 1555 & $15,9 \%$ \\
4. Ind. alberghiera & 742 & $17,3 \%$ & 2090 & $26,4 \%$ \\
5. Servizi pubblici & & & 842 & $4,8 \%$ \\
6. Altri servizi & 1303 & $15,2 \%$ & 2261 & $15,9 \%$ \\
\hline \multicolumn{1}{|c|}{ Terziario } & 2065 & $6,5 \%$ & 11605 & $16,0 \%$ \\
\hline
\end{tabular}

Fonte: Elaborazione dell'autore. Le cifre e percentuali riguardanti il settore terziario sono state ottenute applicando la formula dell'equazione (2) ai coefficienti di localizzazione e la totale dell'impiego nel settore.

I dati e le percentuali della tabella 2 mettono in evidenza il riorientamento verso l'esportazione del settore terziario ticinese, nel corso del dopoguerra. Osserviamo che questo riorientamento si è manifestato in due rami, in particolare, più precisamente nel ramo dei servizi finanziari e in quello dell'industria turistica. In seguito ai miglioramenti della produttività di cui si è detto ma, probabilmente, anche in seguito ad una perdita di importanza del ramo stesso, l'orientamento verso l'esportazione del ramo «trasporti e comunicazioni» è diminuito. Il risultato di queste modificazioni interne si riassume nell'aumento della quota di occupati del settore che si orientano verso le attività di esportazione dal 6,5 al $16,0 \%$.

In conclusione possiamo affermare che, a livello cantonale, il contributo del terziario all'economia ticinese è dato soprattutto dallo sviluppo delle attività del ramo dei servizi finanziari e dallo sviluppo dell'industria alberghiera. In termini di occupazione non si può d'altra parte dimenticare l'espansione del ramo dei servizi pubblici. Tuttavia, come abbiamo dimostrato più avanti, questa espansione è da ricollegare a fenomeni interni al Cantone, quali l'aumento della popolazione, piuttosto che all'esportazione di servizi.

\section{I servizi finanziari e lo sviluppo dell'agglomerato di Lugano}

Per lungo tempo l'attività delle istituzioni finanziarie è stata considerata come un'attività dipendente prettamente legata allo sviluppo delle altre attività dell'economia regionale. Questo perchè le istituzioni finanziarie venivano considerate unicamente nella veste di intermediari tra il risparmio della regione e gli imprenditori desiderosi di investire nella regione stessa. Nel caso della piazza finanziaria di Lugano questa attività di natura tradizionale è stata da lungo tempo superata, per importanza, da quella più moderna di intermediazione tra risparmiatori $\mathrm{e}$ investitori residenti in aree geografiche diverse. Le istituzioni finanziarie diventano in questo caso delle semplici piattaforme girevoli, il cui sviluppo non è più legato all'espansione delle attività reali dell'economia regionale, ma all'evoluzione dei movimenti internazionali di capitale finanziario.

In questa nuova situazione, il ruolo delle attività di intermediazione finanziaria non è più secondario o dipendente, ma diventa indipendente e autonomo rispetto allo sviluppo dell'economia della regione. Come sostenuto dal Polese si può affermare che in una situazione del genere, il ramo delle istituzioni finanziarie diventa l'elemento motore dello sviluppo regionale. Nell'analisi del caso di Lugano non insisteremo sui determinanti storici della situazione particolarmente favorevole allo sviluppo delle attività finanziarie, venutasi a determinare, in questa regione di frontiera, nel corso degli ultimi tre decenni. Rimandiamo chi volesse approfondire l'esame di questo aspetto della problematica dello sviluppo di un agglomerato urbano ad una nostra pubblicazione precedente (ROSSI, 1982). Ricordiamo solamente che nel 1977 si contavano a Lugano 39 banche, delle quali 12 erano state fondate prima del 1956, 16 dal 1956 al 1971 e le restanti 11 durante il periodo dal 1971 al 1977 (FERRARA, RUSCA. 1977). Con le loro filiali, queste banche rappresentavano un centinaio di stabilimenti ai quali occorreva aggiungere un altro centinaio di fiduciarie e una ventina di agenzie di assicurazione.

L'espansione del ramo dei servizi finanziari è stata forte, sopprattutto nel periodo tra il 1960 e il 1975. $\mathrm{Nel}$ corso degli ultimi dieci anni si è notata invece una certa stagnazione, almeno per quanto riguarda le nuove fondazioni e l'aumento dell'occupazione. In termini di cifra di bilancio, invece, il ritmo di sviluppo delle attività finanziarie non ha cessato di progredire.

Per misurare l'impatto delle attività finanziarie sullo sviluppo dell'economia locale accenneremo a quattro tipi di effetti:

a) l'effetto di innovazione

b) l'effetto sull'impiego 
c) l'effetto sul reddito regionale

d) l'effetto sulle altre attività dell'economia dell'agglomerato

\section{a) L'effetto di innovazione}

La letteratura sui processi innovativi tende a distinguere tra due gruppi di innovazione:

- le innovazioni di prodotto

- e le innovazioni di processo.

Secondo noi, lo sviluppo delle attività finanziarie nell'agglomerato di Lugano ha portato sia ad innovazioni di prodotto che ad innovazioni di processo. In un primo tempo, quando per effetto della liberazione dei movimenti internazionali di capitale, gli stabilimenti bancari della zona cominciarono a sviluppare le loro attività internazionali, l'innovazione fu piuttosto un'innovazione di prodotto. Le banche che erano abituate ad operazioni di credito a medio e a lungo termine per le aziende e i consumatori dell'agglomerato, aggiunsero ai loro programmi di attività una nuova serie di operazioni (fondi di investimento esteri, partecipazioni ad emissioni obbligazionarie sui mercati internazionali, finanziamento del commercio internazionale) che erano loro poco conosciute.

Più tardi, il successo stesso delle loro operazioni le portò ad introdurre metodi più moderni ed efficienti di lavoro nelle banche stesse. Si può affermare che le innovazioni di processo caratterizzeranno l'evoluzione dell'attività delle istituzioni finanziarie di Lugano nel corso del prossimo futuro. Le preoccupazioni maggiori sono verso una maggiore efficienza, come pure verso un controllo più oculato delle operazioni.

\section{b) L'effetto sull'impiego}

Dal 1965 al 1980 il numero dei posti di lavoro nel ramo dei servizi finanziari di Lugano è passato da 1814 a 4668. Il grosso dello sviluppo si è manifestato tra il 1965 e il 1975, periodo durante il quale l'impiego di questo ramo è più che raddoppiato. Mentre nel 1965, l'occupazione nel ramo dei servizi finanziari rappresentava il 6,3\% dell'occupazione totale, dieci anni dopo questa quota era salita al 14,3\% . La crescita delle attività finanziarie ha contribuito a mantenere un livello di impiego alto e in espansione in una regione nella quale altri rami di attività e, in particolare, quelli del settore industriale erano in difficoltà. Praticamente si può affermare che nella città di Lugano, durante il decennio 1965-75 per ogni posto perso nel resto dell'economia si creavano due nuovi posti nel ramo dei servizi finanziari. In altri termini l'espansione dell'occupazione in un ramo che non rappresentava che il 6,3\% dell'occupazione totale è bastata per compensare le perdite di posti di lavoro in tutto il resto dell'economia e in- crementare il volume complessivo dell'occupazione. A scanso di equivoci precisiamo che l'effetto sull'impiego non è stato un effetto di tipo moltiplicativo. Non sembra, in altri termini, che l'espansione dell'occupazione nel ramo dei servizi finanziari abbia determinato un aumento del livello di occupazione in altri rami. Si è piuttosto assistito ad una riconversione o ristrutturazione dell'impiego urbano verso il settore terziario. In questo processo il ramo dei servizi finanziari ha svolto, senza ombra di dubbio, il ruolo di ramo-guida.

\section{c) L'effetto sul reddito regionale}

Abbiamo tentato, in altra pubblicazione (ROSSI, 1985) di render conto del possibile effetto dell'importazione di capitali da fuori regione, sottoforma di depositi bancari, sull'evoluzione del reddito della regione di Lugano. Per far questo abbiamo adottato un modello di sviluppo del tipo di quelli della base economica, nel quale oltre alla componente esterna del reddito figura, quale seconda variabile esogena, l'afflusso di mezzi finanziari dall'esterno. In questo modello, quindi, l'equazione per il reddito regionale, che ne rappresenta la forma ridotta, si presenta come segue:

$$
\text { (4) } \mathrm{Y}=\mathrm{a}+\mathrm{bYe}+\mathrm{cDE}
$$

In questa equazione $Y$ rappresenta il reddito regionale, Ye la sua componente esterna e DE l'ammontare dei depositi nelle banche di Lugano di provenienza esterna. La stima di questa equazione per il periodo $1960-75$, con il metodo dei minimi quadrati, ha dato i seguenti risultati:

$$
\mathrm{Y}=\underset{(5,98) \quad 206,679+1,220 \mathrm{Ye}+0,064 \mathrm{DE} \quad \mathrm{R}^{2}=0,983}{(16,79) \quad(0,35)}
$$

In parentesi sono riportati i valori del rapporto tra la stima del parametro e il suo errore sistematico, coefficienti che servono per valutare il grado di significatività del parametro. Stando a questi valori, la variabile $\mathrm{DE}$, che, lo ripetiamo, rappresenta i depositi esterni, non sarebbe significativa. In altri termini i depositi esterni nelle banche della piazza di Lugano non sembrerebbe avere un influsso apprezzabile sull'evoluzione del reddito regionale. Questa influenza limitata dei depositi esterni deve essere attribuita al fatto che gli stessi non sono investiti nell'economia della regione, ma, in buona misura, collocati fuori della stessa sui mercati finanziari di tutto il mondo. Utilizzando le equazioni del nostro modello della base economica e i parametri stimati dell'equazione (5) abbiamo infatti potuto stimare che circa il $70 \%$ di tutti i depositi affluiti nelle banche di Lugano (in provenienza dall'esterno o dall'interno della regione) vengono ricollocati fuori della 
regione luganese. Evidentemente, da questo profilo, il ruolo delle banche, che pur controllano e muovono somme ingenti di capitali, non è molto determinante (ROSSI, 1985).

\section{d) L'effetto sulle altre attività della regione}

Attraverso le loro spese correnti, le aziende del ramo dei servizi finanziari possono contribuire allo sviluppo di altre aziende della regione. Per poter accertare l'importanza di questi effetti di retroazione ocorrerebbe poter analizzare le relazioni interindustriali del ramo dei servizi finanziari, operazione quanto mai difficile, per la mancanza quasi assoluta di informazioni. Dai dati in nostro possesso, raccolti tra l'altro a mezzo di un'indagine svolta presso alcune banche della piazza di Lugano, possiamo avanzare le seguenti osservazioni. Nel 1980, la regione di Lugano nella quale sono compresi, oltre la città e i comuni dell'agglomerato, i comuni di due regioni di montagna, per un totale di 90 istituzioni locali e circa 100000 abitanti, disponeva di un reddito di 1707,1 milioni di franchi (FISCHER, RUTISHAUSER, BAUMELER, 1983). L'importanza degli effetti di retroazione del ramo dei servizi finanziari sarà calcolata rispetto a questa somma.

\section{Salari e stipendi}

La somma dei salari e degli stipendi versati dalle aziende del ramo finanziario è stata stimata partendo da uno stipendio medio che, nel 1980, era di circa 30000 franchi. Tenendo conto del numero dei posti di lavoro del ramo nella regione (a quelli della città occorre aggiungere i posti di lavoro negli altri comuni della regione) abbiamo potuto stimare in circa 180 milioni di franchi la somma annualmente versata degli istituti finanziari della regione. Questa somma rappresentava, nel 1980 , il $10,5 \%$ del reddito disponibile e il $15 \%$ del reddito versato ai lavoratori dipendenti. Si tratta di quote certamente non disprezzabili.

\section{Acquisto di beni e servizi}

Anche in questo caso abbiamo cercato di stimare una quota per posto di lavoro, partendo dai dati riguardanti le spese generali che figurano nei conti delle banche. Seguendo questo metodo siamo arrivati a stimare, per il 1980, una somma di 122 milioni di franchi, spesi per l'acquisto di beni e servizi. Se da questo totale togliamo le spese che interessano acquisti eseguiti fuori regione, ci accorgiamo che la spesa degli istituti finanziari per l'acquisto di beni e servizi nella regione corrisponde approssimativamente all' $8 \%$ del reddito regionale. Si tratterebbe quindi di un effetto meno importante di quello attribuibile ai salari e agli stipendi versati da queste istituzioni.
Osserviamo da ultimo che, per mancanza di dati, non abbiamo potuto stimare, nemmeno per approssimazione, l'importanza degli investimenti eseguiti dagli istituti finanziari nell'economia regionale. $\dot{E}$ probabile che, specie in relazione agli investimenti nelle costruzioni, questo contributo sia notevole. In conclusione, analizzando sulla base dello schema degli effetti molto semplice, presentato in questa parte del nostro contributo, l'impatto del ramo dei servizi finanziari sull'economia urbana e della regione di Lugano, abbiamo potuto renderci conto che esso è molto importante dal profilo dell'occupazione e dal profilo dei redditi distribuiti in relazione al rapporto di impiego. Esso è meno importante dal profilo del contributo che i depositi affluiti dall'esterno potrebbero dare al processo di accumulazione dell'economia regionale come pure dal profilo del contributo che gli istituti finanziari possono dare allo sviluppo di altre aziende, attraverso i loro acquisti di beni e servizi. In relazione a questo ultimo punto dobbiamo tuttavia ammettere che le nostre conclusioni sono state dedotte da un esame estremamente sommario della situazione esistente in materia di relazioni interindustriali.

\section{Conclusioni}

Il Cantone Ticino ha conosciuto, nel corso degli ultimi trentanni una decisa riconversione della sua struttura di produzione, dominata dall'espansione delle attività del settore terziario. Questa espansione ha potuto realizzarsi da un lato in seguito al crescere della domanda di servizi di natura pubblica. In questo senso, l'espansione del terziario deve essere considerata come una conseguenza del migliorato livello di benessere e, quindi, in ultima analisi dello sviluppo dell'economia. In relazione ad altri rami del terziario, tuttavia, l'espansione dell'occupazione ha avuto carattere più autonomo, rispondendo, come abbiamo cercato di dimostrare nel paragrafo 2 del nostro contributo, ad una domanda crescente di servizi in provenienza dall'esterno. L'esportazione di servizi si è sviluppata soprattutto in due rami:

- il ramo dell'industria alberghiera

- il ramo dei servizi finanziari.

Nel paragrafo 3 del nostro articolo abbiamo cercato di illustrare i possibili effetti positivi dell'espansione del ramo dei servizi finanziari in un'economia urbana, l'economia della città e della regione funzionale urbana di Lugano. Ovviamente l'evoluzione così rapida dei servizi finanziari, servizi che, specie in relazione alle operazioni internazionali, si accompagnano spesso ad un alto grado di rischio, non ha avuto, nemmeno dal punto di vista dei soli effetti 
economici, solamente aspetti positivi. Occorre tuttavia insistere su quest'ultimi perchè spesso, sull'onda delle proteste sollevate da un piccolo o grosso scandalo bancario, si tende a minimizzarli per non dire a dimenticarli completamente. Lugano e la sua regione, ma anche altri centri e regioni del Ticino, hanno saputo trarre profitto dall'espansione del ramo dei servizi finanziari. I problemi creati da questo sviluppo non sono da sottovalutare ma restano, li si consideri da qualunque prospettiva lo si voglia, problemi di sviluppo. Per un'economia di regione periferica, come è, e resterà, quella del Canton Ticino è preferibile, reputiamo, occuparsi con le difficoltà poste dallo sviluppo, piuttosto che con i problemi sollevati dalla crisi.

\section{Le secteur tertiaire et le développement régional: le cas tessinois}

Dans la littérature de science régionale plus récente, on attribue au secteur tertiaire un rôle de plus en plus important, en tant que moteur du développement régional. Cette activité motrice se réaliserait par l'exportation de services. Dans notre contribution on essaie de vérifier l'importance de l'apport du tertiaire au développement de l'économie du Canton Tessin par deux types d'approches différentes:

- la première, au niveau cantonal, consiste à mesurer les modifications temporelles des coefficients de localisation et à apprécier, par la suite, les modifications dans la part de l'emploi des branches du tertiaire qui est orientée vers l'exportation

- la deuxième, au niveau d'une agglomération, se résume dans la tentative d'apprécier les effets induits du développement d'une des branches mo- trices du secteur tertiaire tessinois: les services financiers.

La conclusion qu'on peut tirer de cette étude est que le tertiaire est un secteur très important surtout du point de vue de l'évolution de l'emploi.

\section{Note bibliografiche}

CHRISTALLER, W. (1933): Die zentralen Orte in Süddeutschland, Jena.

DUPASQUIER, J.-N. (1984): Elaboration et analyse de la statistique de l'emploi public en Suisse, 1950-1980, Cahiers du Département d'Econométrie, Ginevra.

FERRARA, C./RUSCA, G. (1977): Le centre de Lugano: les facteurs qui ont déterminé la modification de son organisation spatiale et les conséquences, Ginevra.

FISCHER, G./RUTISHAUSER, P./BAUMELER, J. (1983): Räumliche Einkommensdisparitäten in der Schweiz: das persönlich-verfügbare Einkommen nach Regionen, 1970 und 1980, Programma nazionale di ricerca "Problemi regionali", Rapporti dei lavori, no 40, Berna.

ISSERMAN, A. (1977): Alternative Economic Base Bifurcation Techniques: Theory, Implementation, Results. In: S. PLEETER: Economic Impact Analysis, Methodology and Applications, Boston, pp. 32-53.

MERLIN, P. (1973): Méthodes quantitatives et espace urbain, Parigi.

POLESE, M. (1974): Le secteur tertiaire et le développement régional. Vers un modèle des activités motrices. In: L'actualité économique, 50, no 4, pp. 475-90.

ROSSI, A. (1982): La regione funzionale urbana di Lugano, Zurigo-Bellinzona.

ROSSI, A. (1985): Regionale Disparitäten, Vorlesungsskript, Zurigo-Losanna.

ROSSI, A. (1985): Lo sviluppo dell'agglomerato e l'attività delle autorità locali, Losanna. 